\title{
La función de producción Cobb Douglas y su aplicación en la economía boliviana
}

\section{The Cobb Douglas production function and its application in the Bolivian economy}

Pedro Feraudi Gonzales

Universidad Técnica de Oruro, Bolivia

Dante Ayaviri Nina

Universidad Nacional de Chimborazo, Ecuador

Autor corresponsal: pedroferaudi@gmail.com, vdayaviri@gmail.com

Fecha de recepción: 09 de Octubre de 2017 - Fecha de aceptación: 15 de Abril de 2018

Resumen: El presente estudio tiene por objetivo aplicar la función de producción para la economía boliviana determinando la relación existente entre las variaciones de la producción como consecuencia de las variaciones de los factores de producción capital y trabajo. Para ello, se aplica la función de producción de Cobb Douglas. El análisis económico permite determinar las relaciones de la formación bruta de capital fijo y la población ocupada que contempla una serie de tiempo del periodo 1985-2015; por otra parte, se ha recurrido a la literatura especializada e investigaciones desarrolladas en la temática. Los resultados de la investigación, determinan una relación directa y significativa entre la producción, capital y trabajo. En consecuencia, la función de producción planteada es un método que permite determinar el aporte de cada uno de los factores productivos al proceso de crecimiento y comportamiento económico de Bolivia.

Palabras Claves: función de producción; economía; Coob Douglas; crecimiento económico

\begin{abstract}
The purpose of this study is to apply the production function for the Bolivian economy by determining the relationship between production variations as a consequence of changes in capital and labor factors of production. To do this, the production function of Cobb Douglas is applied. The economic analysis allows to determine the relations of the gross formation of fixed capital and the occupied population that contemplates a series of time of the period 1985-2015; On the other hand, specialized literature and research on the subject have been used. The results of the research determine a direct and significant relationship between production, capital and labor. Consequently, the production function proposed is a method that allows determining the contribution of each of the factors of production to the process of growth and economic behavior of Bolivia.
\end{abstract}

Key Words: production function; economy; Coob Douglas; economic growth 


\section{Introducción}

La función de producción es un elemento importante en los procesos productivos de bienes y servicios (Kumbhakar et al., 1991; Bellod (2011), Jehle y Reny, 2011; Wang y Fu, 2013; Ilca y Popa, 2014; González et al., 2004; Cheng y Han, 2014), el origen fue dado "por Wicksteed (1894) en el campo de la microeconomía supuso una revolución en la Teoría de la Producción y la Distribución. Trabajos posteriores permitieron popularizar formas específicas como la Función Cobb - Douglas [Cobb y Douglas (1928), Douglas (1948)], la Función de Leontief [Cassel (1918), Leontief (1941)], la Función de Elasticidad de Sustitución Constante (CES) [Arrow et al (1961)]" citado en (Bellod, 2011, p.10). En esa línea, López (2015), señalan que la función de producción permite realizar análisis de variables complejas de la economía; y por otra parte, Parra (2012), indica que la importancia radica en el conocimiento y la tecnología de la producción y de los rendimientos a escala pueden considerarse mediante enfoques diferentes entre los que señala dos como: el enfoque de los costos y el producción; en cambio Sancho (2003) y Jesús y McCombie (2005, afirman que una función de producción debe proporcionar información de procesos productivos y sus consecuencias. Sin embargo, existen todavía lagunas importantes en el conocimiento de estos procesos (Díaz y Giral, 2011; MuineloGallo, 2012). Autores como Díaz y Giral (2011), Cubel y Palafox, (2002) y Castellanos et al., (2006), parten de la búsqueda de organizar los diferentes factores relacionados con la producción a partir del modelo de Cobb Douglas, que influyen en el crecimiento de los países.

Existen varios trabajos realizados en la aplicación del modelo de producción de Cobb Douglas, en el sector de infraestructura (Boscá et al., 2002; Nombela, 2005; Zurita et al., 2016; ), cultura (Parra, 2012), sector financiero (Ramírez, 2015; Felipe y Adamas, 2005), agropecuaria (López y Palomares, 1999; Martinez y Castillo, 1987; Cortazar y Montaño, 2011) informática (Parag, 2008; Díaz et al., 2013), económico (Cubel y Palafox, 2002; Bellod, 2011; Castellanos et al., 2006; Arango, 1993; Gonzalez et al., 2004; Montoya y Soto, 2011; Cheng y Han, 2014; Dixit y Stiglitz, 2004) y otros. Los debates más importantes se concentran en la teoría económica, los fundamentos teóricos del concepto de función de producción agregada y las implicaciones de esta, en particular como base de una teoría de distribución del producto agregado. Castellanos et al., (2006) y Rajimon (2010) indican que es importante su abordaje porque permiten modelar funciones de producción para buscar relaciones entre las causas y sus efectos en los procesos productivos y económicos.

En el periodo 1980-1985 la economía boliviana sufrió una recesión, el Producto Interno Bruto (PIB) fue el más bajo de América Latina; entre las demandas y necesidades urgentes fue reactivar la producción y el empleo. Así, a fines de 1984 y principios de 1985, la situación económica boliviana fue considerada como una de las más frágiles de América Latina. Por lo que, la orientación de las políticas públicas fueron orientadas a atender las necesidades de incentivo y crecimiento económico. Fue de esta forma, en el periodo 1985-2005 se aplica el Programa macroeconómico de ajuste estructural, cuyos lineamientos y recomendaciones fueron planteados por el Fondo Monetario Internacional y el Banco Mundial (Durán, 2015; Rivera, 2016). Así, con el programa de ajuste estructural se redujeron las tasas de inflación y un incremento leve en el PIB; a su vez, la promoción de la Inversión Extranjera Directa IED, como política de Estado. De acuerdo con Humerez - Dorado (2004:11), en la década de los ochenta, la actividad económica apenas creció a una tasa promedio de $0.16 \%$, esto debido principalmente a 
la crisis registrada entre los años 1982 y 1986, que desalentó la inversión productiva y favoreció las actividades especulativas. En consecuencia, el PIB per cápita registró una tasa de crecimiento negativa, alcanzando un promedio anual de $-2.13 \%$, mientras que el consumo solo creció en $0.43 \%$, revelando una situación de fuerte deterioro de la condición de vida de la población boliviana. Desde los noventas hasta el 2004 se observa un periodo de moderado crecimiento expresado en una tasa promedio de $3.41 \%$, resultante de la aplicación de procesos de reformas estructurales, siendo las más importantes las denominadas de 'primera generación', concentrada en la implementación de medidas destinadas a establecer el mercado como mecanismo de asignación de recursos en la economía y, de 'segunda generación', compuesta por un conjunto de medidas orientadas a remover restricciones estructurales. El mayor crecimiento relativo en este periodo, se ha reflejado también en un mejoramiento modesto del bienestar de la población, el PIB per cápita creció a una tasa anual de $1.01 \%$, mientras que el consumo creció a una tasa promedio de $3.09 \%$.

Por otra parte, se puso en marcha la política de desgravación tributaria y desregulación de los mercados. La privatización y capitalización de las empresas públicas permitió mayor oportunidad de inversión del sector privado, especialmente en el sector de hidrocarburos, telecomunicaciones y energía (Aguirre, 2001). Según Calderón, (1999) y Morales (2014), la economía boliviana podría haber alcanzado un mejor crecimiento si la crisis no habría afectado a los sectores productivos, la apreciación excesiva de la moneda, que desincentivó las exportaciones, y la caída de las remesas. De esta forma, en el entorno macroeconómico, Bolivia comienza a deteriorarse, contribuyendo a un crecimiento promedio anual del PIB cercano al 2 por ciento durante el periodo 1999-2003, y en el periodo 2004-2007 fue moderado y alcanza un 4.2\%; en tanto que el promedio latinoamericano se sitúa por encima del 5.5\% (Durán, 2015), el caso del Perú fue de $8 \%$. En este contexto, la presente investigación pretende aproximarse al estudio de la economía boliviana a través de la aplicación de la función de producción, intenta determinar la relación entre las variaciones de la producción como consecuencia de las variaciones de los factores de producción capital y trabajo para el periodo 1985-2015, a partir de las variables formación bruta de capital fijo y la población ocupada.

\section{Métodos y materiales}

El método que permite realizar el análisis de la economía boliviana, está basado en el modelo de función de Producción de Coob Douglas. Algebraicamente una función de producción puede escribirse de la forma:

Donde:

$$
Y_{t}=f\left(K_{t}, L_{t}\right)
$$

$\mathrm{Y}_{\mathrm{t}}=$ Representa la cantidad de bienes y servicios que produce una economía en el tiempo $\mathrm{t}$, es la Variable dependiente de las dotaciones o de los acervos existentes de los factores de capital y de trabajo $\mathrm{K}_{\mathrm{t}}=$ Representa el factor capital en el tiempo t, variable independiente $\mathrm{L}_{\mathrm{t}}=$ Representa el factor trabajo en el tiempo t, variable independiente 
Es una de las funciones de producción más empleadas en el ámbito de la economía, basada su aplicación en el manejo y cumplimiento de las propiedades básicas. Se debe su existencia a Paul Douglas y Charles Cobb en 1927. La función explicada por Cobb Douglas es:

$$
Y_{t}=A K_{t}^{\alpha} L_{t}{ }^{B} ; \quad \text { Donde: } 0<\alpha, \beta<1
$$

$Y_{t}=$ Producción

$A=$ Progreso Técnico Exógeno

$K_{t}=$ Stock de Capital

$L_{t}=$ Número de empleados en una economía

En esta función formalizada por Cobb Douglas, $\alpha$ y $\beta$ son los parámetros que representan el peso de los factores $\mathrm{K}$ y L en la distribución de la renta. A, es el progreso Técnico o Productividad total de los factores que es una variable no directamente observable, pues representa un estado no cuantificable formado por factores tales como: la organización empresarial, los conocimientos de los empresarios y trabajadores o el nivel de aplicación de tecnología. Por tanto, esta función de producción está compuesta por dos factores productivos que se mantienen constantes en el tiempo y un factor adicional que es el progreso técnico. La idea de la función de producción es muy intuitiva, pues representa combinaciones de los factores capital y trabajo que satisfacen las propiedades:

- Rendimientos constantes a escala, homogeneidad de grado 1. Es decir, si el capital y el trabajo se incrementan en la misma proporción, la producción aumentará también en la misma proporción.

- Productividad marginal positiva y decreciente. Esta función es la que introduce el postulado más básico de la economía clásica, los rendimientos marginales decrecientes, tanto de capital como de trabajo.

La función de producción de Cobb-Douglas parte de una idea básica, la cual reside en que la producción de una economía depende fundamentalmente de sus dotaciones de capital y de trabajo, esto quiere decir que, si en una economía se quiere producir más, requiere emplear una cantidad de capital y una cantidad de trabajo tales que le permitan generar producto (Díaz et al., 2013; Cortazar y Montaño, 2011; Ilca y Popa, 2014; Wang y Fu, 2013; Cheng y Han, 2014). Si en la economía las empresas requieren producir bienes y servicios, entonces requerirán emplear unas cantidades de capital y unas cantidades de trabajo, la versión más sencilla del modelo Cobb Douglas es explicada por un coeficiente técnico de producción que se denomina A, que representa el grado de conocimiento, de desarrollo, de investigación, que está al servicio de la actividad productiva. A, en la literatura microeconómica es el coeficiente técnico de producción y es un elemento fundamental que contribuye a la producción de bienes y servicios, la producción depende del factor $\mathrm{K}$ y la incidencia que ejerce este factor sobre la capacidad productiva se ve reflejada en un coeficiente denominado alfa $\alpha$,

$$
Y=A K^{\alpha} L^{\beta}
$$

La relación que existe entre el coeficiente $\alpha$ y K es que $\alpha$ es un exponente que recoge la incidencia que el factor $\mathrm{K}$ ejerce sobre la producción $\mathrm{Y}$, si por un momento imaginamos lo que 
sucedería en una economía cuando se incrementa la cantidad de $\mathrm{K}$ o como en la literatura microeconómica se establece el stock de capital. Lo que se espera es que la capacidad productiva de las empresas aumente también el coeficiente $\propto$ que recoge la incidencia del impacto. La teoría dice que este coeficiente

$\boldsymbol{\alpha}$ se denomina productividad marginal del capital

$$
\begin{gathered}
\propto=\frac{\partial Y}{\partial K} \\
\propto>0 \\
\propto<1
\end{gathered}
$$

Asimismo, una economía requiere dotaciones de trabajo, que se denominan con la letra $\mathrm{L}$ y así como sucedía con el factor K, el factor L tiene incidencia, tiene un efecto sobre la producción. Si las cantidades de trabajo expresadas en horas o en número de trabajadores varían, por ejemplo, se incrementan, lo que se esperar es que en alguna medida la producción también se incremente y lo que haremos es insertar un coeficiente $\beta$ que permita calcular el efecto que genera una variación en la producción explicada por una variación en el factor L; la teoría dice también que eso lo medimos a partir del coeficiente Beta $\beta$, entonces el coeficiente $\beta$ mide el cambio que genera el factor L sobre la producción y es lo que en la literatura microeconómica se conoce con el nombre de:

$$
\begin{gathered}
\boldsymbol{\beta} \text { se denomina productividad marginal del trabajo } \\
\beta=\frac{\partial Y}{\partial L} \\
\beta>0 \\
\beta<1
\end{gathered}
$$

Donde, $\alpha$ es un coeficiente de productividad y particularmente es un coeficiente de productividad que mide el efecto que tiene una variación en el capital cuando ella afecta a la producción, en términos del cálculo diferencial se dice que el coeficiente $\alpha$ es igual a lo que podríamos denominar como una derivada, un cociente de derivada que explica cuál va a ser la variación que se experimente en la producción debido a una variación generada en el factor en este caso $\mathrm{K}$, entonces el coeficiente $\alpha$ simplemente es un coeficiente de productividad que dice que si se modifica cambia o varia el factor $\mathrm{K}$, en qué proporción o en qué medida variará la cantidad producida $Y$, se entiende que $\alpha$ mide las variaciones que se dan en la producción, debidas a las variaciones que se dan en el factor $\mathrm{K}$, una forma simple de entender este indicador es pensar que el denominador explica al numerador, así el coeficiente $\alpha$ simplemente dice que si se varía el factor $\mathrm{K}$, producirá una variación en $\mathrm{Y}$, a esto se llama productividad marginal del factor $\mathrm{K}$, se dice que esa productividad es marginal porque cuando aumentamos las cantidades de $\mathrm{K}$, es cierto que aumente la $\mathrm{Y}$; tendremos una relación positiva es decir que a mayor volumen de $\mathrm{K}$ se espera un volumen mayor de $\mathrm{Y}$, pero el hecho de que sea marginal implica que el aporte o la contribución que el factor $\mathrm{K}$ le hace a la producción $\mathrm{Y}$, cada vez es menos proporcional y por lo tanto si bien advertimos que el indicador de productividad es positivo eso significa que es mayor que cero y la teoría sostiene que es menor que uno. 


$$
\begin{aligned}
& \propto>0 \\
& \propto<1
\end{aligned}
$$

Si $\alpha$ es un indicador de productividad, podríamos decir que $\beta$ es también un indicador de productividad, pero en este caso $\beta$ mide la capacidad que la producción tiene para producir bienes dependiendo de las variaciones que se generen en el factor L, es decir que la productividad del trabajo no es otra cosa que la relación existente entre las variaciones que se generan en la producción debido a las variaciones que se dan en el factor trabajo, por tanto, a mayores cantidades de trabajo se espera mayores cantidades de producto, pero al advertir que la relación es una relación marginal se advierte que la contribución del factor trabajo que hace a la producción es cada vez menos que proporcional. Si se suman por ejemplo el valor del coeficiente alfa que define la productividad del factor capital más el coeficiente beta que define la productividad del factor trabajo y esa suma será igual a uno, entonces podríamos decir que las empresas tienen rendimientos constantes a escala, eso significa que si duplicamos el esfuerzo en capital y trabajo, la producción también se duplica, es decir todo esfuerzo en capital y trabajo será un esfuerzo recompensado en la capacidad productiva de la empresa, esa situación implica que la firma tiene rendimientos constantes a escala,

$$
\propto+\beta=1 \text { rendimientos constantes a escala }
$$

Una segunda situación podría derivarse de que la productividad del factor capital sumada a la productividad del factor trabajo nos dé un coeficiente superior a uno, en cuyo caso lo que tendríamos es que esta empresa se caracteriza por tener rendimientos crecientes a escala, eso significa que a un esfuerzo en las dotaciones en capital y trabajo, el rendimiento en la producción será más que proporcional a ese esfuerzo. Por ejemplo, si duplicamos el esfuerzo en capital y trabajo, el resultado será más del cien por ciento, en este caso existen rendimientos crecientes a escala,

$$
\propto+\beta>1 \text { rendimientos crecientes a escala }
$$

Ahora bien, si se considera la suma de coeficientes de productividad del capital sumada a la productividad del trabajo, el resultado será un valor menor que la unidad, entonces nos acercaremos a los rendimientos decrecientes a escala, lo que significa que si duplicamos los esfuerzos productivos en capital y trabajo, la producción se incrementa en una proporción inferior a los esfuerzos realizados en capital y trabajo.

$$
\propto+\beta<1 \text { rendimientos decrecientes a escala }
$$

La función de producción requiere ser linealizada, es decir, que para hacer un ejercicio de estimación de los coeficientes alfa y beta, se debe tener los coeficientes u exponentes como factores lineales, por ejemplo si quisiéramos encontrar o estimar el valor de los coeficientes, la teoría señala que deben ser lineales, uno de los convencionalmente utilizados para estimar esos coeficientes alfa y beta es el método conocido como el de Mínimos Cuadrados Ordinarios Matriciales,

$$
\begin{gathered}
Y=A K^{\propto} L^{\beta} \\
\ln Y=\epsilon^{A}+\propto \ln K+\beta \ln L
\end{gathered}
$$


es un método de estimación económica, las estimaciones puntuales que en este caso serían alfa y beta requerirán ser lineales, una forma de linealizar esta ecuación es bajo luz de instrumentos matemáticos de suavización como los logaritmos entonces para linealizarla se explica la producción como suavizada a partir de los logaritmos naturales, eso significa, que si el logaritmo de Y $(\ln Y)$. Finalmente, el mismo tratamiento se aplica al coeficiente beta que tiene la forma lineal y que multiplica o pondera las variaciones generadas en el factor trabajo, de esta manera la producción suavizada a partir de logaritmos es explicada por el capital suavizado también en términos logarítmicos y por el factor trabajo suavizado en términos logarítmicos, y el coeficiente alfa y beta son coeficientes de productividad que miden el efecto que generan las variaciones de capital o las variaciones de trabajo sobre la producción, un aspecto a tomar en cuenta, es que el indicador alfa se constituya en uno de los primeros indicadores de rentabilidad dado que indica cuál es la respuesta que la producción sufre cambios en el capital.

\section{El modelo}

\section{Resultados}

El modelo econométrico plantea contrastar empíricamente la función de producción de Cobb Douglas para la economía boliviana en el período 1985 - 2015, la relación de comportamiento de la ecuación a estimar es:

$$
Y_{t}=A^{*} K_{t}^{\alpha} * L_{t}^{B} * e^{u_{t}}
$$

Para la estimación del modelo econométrico por el método de Mínimos Cuadrados Ordinarios (MCO) se debe partir de una función lineal en los parámetros, y como la función de Cobb Douglas no cumple con esta condición, se debe linealizar la ecuación aplicando logaritmos:

$$
\log Y_{t}=\log A+\alpha \log K_{t}+B \log L_{t}+u_{t}
$$

Donde:

$Y_{t}=$ Producción del t-ésimo año, la serie realacionada es el Producto Interno Bruto Real en miles de dólares a precios de 2010 (CEPAL, 2015). Comisión Económica para América Latina y el Caribe - Estimaciones propias con base en fuentes oficiales del INE - Bolivia 1990 - 2015.

$$
K_{t}=\text { Stock de Capital del t-ésimo año, la serie relacionada es la Formación Bruta de Capital }
$$
Fijo Real en miles de dólares a precios de 2010 (CEPAL, 2015). Estimaciones propias con base en fuentes oficiales del INE - Bolivia 1990 - 2015

$L_{t}=$ Empleo del t-ésimo año, la serie relacionada es la Población ocupada en miles de personas; Fuente: División de Población de Naciones Unidas. Perspectivas de la población mundial; Naciones Unidas, Departamento de Asuntos Económicos y Sociales (Banco Mundial, 2016) en su Serie 1960 -2015, con las estimaciones propias con fuentes oficiales de INE - Bolivia. Así, la estimación del modelo por el método mínimos cuadrados ordinales se observa en el gráfico 1. 


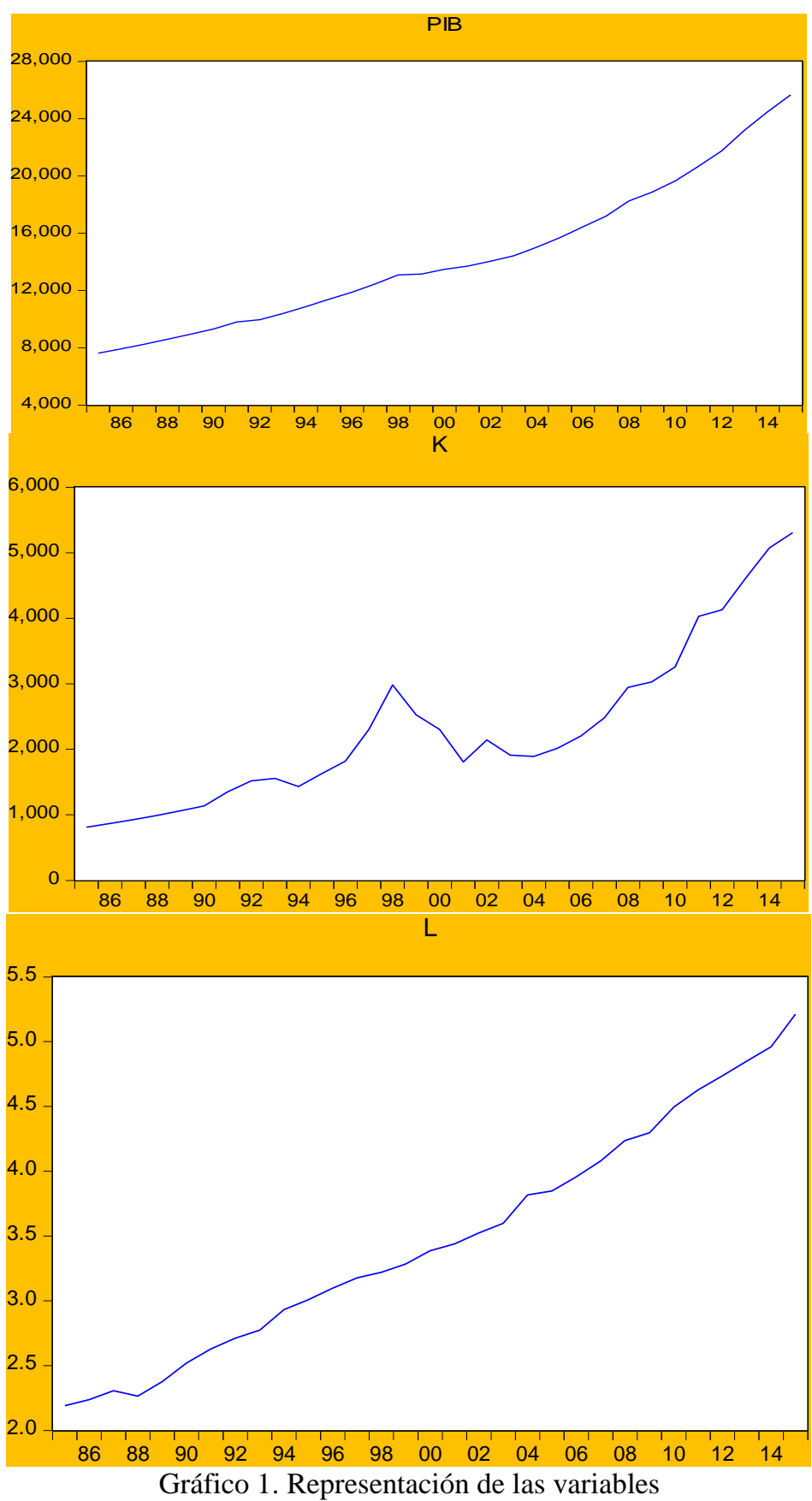




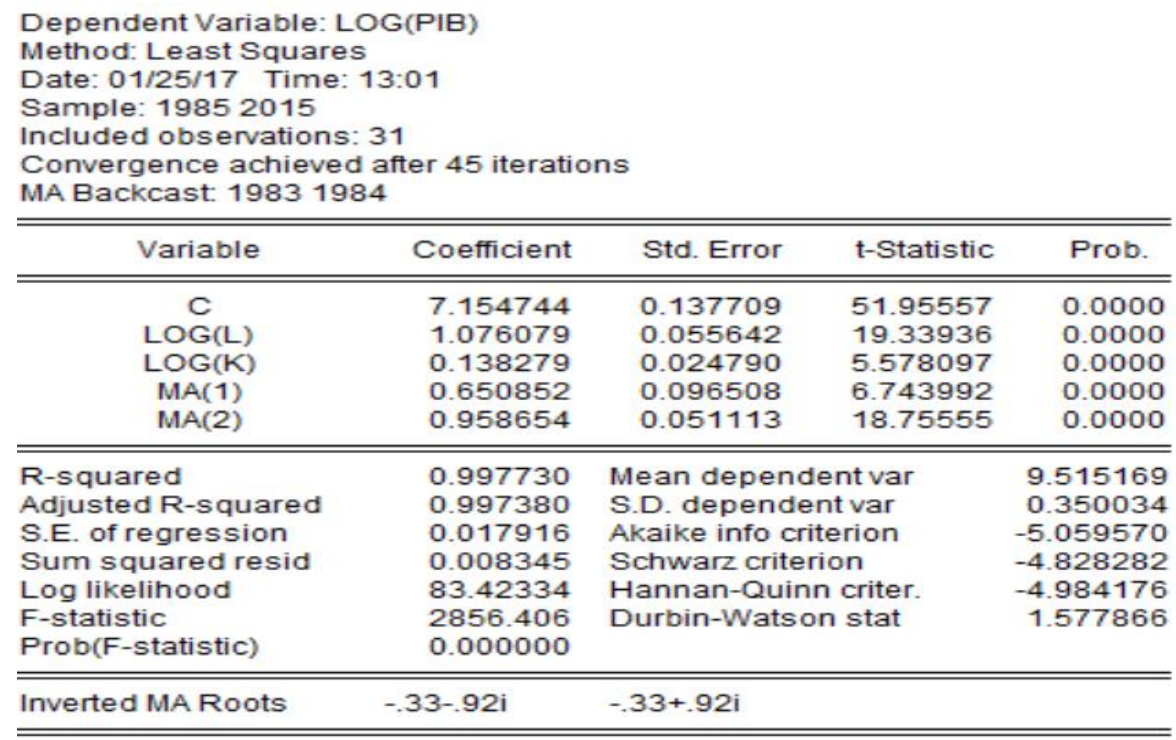

Gráfico 2. Estimación del modelo

Fuente: Elaboración propia

Siendo la ecuación estimada:

$$
\log (P I B)=7.154744+1.076079 * \log (L)+0.138279 * \log (k)
$$

\section{Análisis e interpretación del modelo estimado con bondad de ajuste}

En base a la estimación de la función de producción de Cobb Douglas para la economía boliviana para el periodo 1985 - 2015, se observa que el coeficiente de determinación indica que el 99,77 por ciento de las variaciones que ocurren en la Producción total de Bolivia se explican por las variaciones de la Formación Bruta de Capital Fijo y la Población Ocupada. Asimismo, se contrasta la hipótesis: $\mathrm{H}_{0} \alpha=\beta=0$ con el objeto de verificar si todas las variables exógenas incluidas en el modelo son significativas conjuntamente; en este sentido se compara el valor del estadístico "F" de Fisher calculado en la tabla de salida del Eviews que es igual a 2856.4 lo cual indica que la Formación Bruta de Capital Fijo y la Población Ocupada conjuntamente tienen efecto significativo o una relación directa o positiva sobre la producción final de bienes y servicios en Bolivia.

\section{Análisis individual de los parámetros estimados}

El estadístico “t” de Student calculado de la tabla de salida del Eviews ayudará a verificar si las variables exógenas incluidas en el modelo son individualmente significativas para explicar a la variable endógena; como se aprecia el estadístico " $t$ " para las variables LOG(K) y LOG(L) es 5.58 y 19,33 respectivamente, entonces se acepta la hipótesis alternativa de que las variables exógenas Formación Bruta de Capital Fijo y Población Ocupada tienen efecto individual significativo sobre la Producción total de Bolivia. Por otro lado, se aprecia también que la Población Ocupada es la variable que tiene mayor efecto positivo sobre la Producción en la economía boliviana. 
Los signos de los parámetros estimados indican la relación que existe entre las variables exógenas y la variable endógena; así se observa una relación directa positiva entre el Stock de Capital y la Producción lo que significa que un aumento en el Stock de Capital incrementará los niveles de Bienes y Servicios finales producidos en Bolivia. De igual manera, la Población Ocupada y la Producción tienen una relación positiva, lo cual implica que si la Población Ocupada se incrementa la Producción también sufrirá un incremento.

\section{Discusión}

En este epígrafe se plantea algunas cuestiones relacionadas al comportamiento de las elasticidades. Siendo $\alpha: 0,138 \rightarrow$ es la elasticidad Producción - Capital, indica que si la Formación Bruta de Capital Fijo se incrementa en 7.5\% en la ecuación estimad, la producción se incrementará en 1,035\%, lo cual refleja la baja incidencia del Capital sobre la Producción; $\beta$ : $1.076 \rightarrow$ es la elasticidad Producción - Trabajo, e indica que si la Población Ocupada se incrementa en $2 \%$, la producción boliviana se incrementará en $2.15 \%$; por tanto, significa que la población ocupada tiene una incidencia significativa en la producción de Bolivia. Así, $\alpha+\beta$ :

$(0.14+1.08)=1.22 \rightarrow$ La suma de las elasticidades del factor Capital y Trabajo indica el tipo de rendimiento que muestra la función de producción de Cobb Douglas para el caso de la economía boliviana considerando el período 1985 - 2015, se observa que el resultado es mayor a uno, entonces se tiene rendimientos crecientes, esto quiere decir que si aumenta los factores de producción capital y trabajo en un determinado porcentaje, la producción también se incrementaría en esa medida. La pregunta es, ¿cómo afectaría la disminución ambos factores en la producción?

\section{Conclusiones}

La función de producción de Cobb Douglas estimada para la economía boliviana, establece que el $99,77 \%$ de las variaciones que ocurren en la producción total, se explica por las variaciones en la formación bruta de capital fijo y la población ocupada en el periodo analizado. Por otra parte, la baja incidencia del capital sobre la producción se debe principalmente a que la economía boliviana, está centrada en el sector primario, como ser la producción de materias primas y consecuentemente la comercialización en mercados externos; en tanto que la industrialización de las mismas ha quedado rezagada, sin que se genere un valor añadido. Finalmente, se contrasta que la elasticidad Producción - Trabajo es mayor a la elasticidad Producción - Capital, en consecuencia, la población ocupada es la variable que tiene mayor efecto positivo sobre la producción en la economía boliviana.

\section{Bibliografía}

Arango, H. (1993). Ecuación de la frontera de posibilidades de producción utilizando la función Cobb-Douglas, Lecturas de Economía, № 39, págs. 151-182.

Aguirre, A. (2001). La deuda externa y el programa HIPC en Bolivia. CEDLA

Banco Mundial (2016). Estadísticas, Población total en Bolivia 2016 Grupo Banco Mundial. Reservados todos los derechos. Washington, USA. 
Bellod Redondo, J. F. (2011). La función de producción Cobb Douglas y la economía española, Revista de economía crítica, $\mathrm{N}^{\circ} 12$, págs. 9-38.

Boscá, J.E.; Escribá, J. y Murgui, M.J. (2002). The Effect of Public Infrastructure on the Private Productive Sector of Spanish Regions, Journal of Regional Science, No 42, Vol 2, págs. 301-326.

Calderón, F. (1999). El nuevo Estado y la integración social. Reflexiones a partir de la experiencia boliviana, Nueva sociedad, $\mathrm{N}^{\circ} 159$, págs. 87-95.

Castellanos Pérez, M.; Martínez Garza, Á.; Colmenares, B.; Martínez, M.; Rendón Sánchez, G. (2006). Región confidencial para el óptimo económico de una función de producción CobbDouglas. Agrociencia, Vol 40, N 1, págs. 117-124.

CEPAL (2015). Cepalstat. Estadísticas E Indicadores Económicos. Cuentas Nacionales anuales en Dólares. Producto Interno Bruto (PIB). Santiago de Chile.

Cheng, M. L., \& Han, Y. (2014). A modified Cobb-Douglas production function model and its application. IMA Journal of Management Mathematics, Vol 25, Nº 3, págs. 353-365.

Cortazar, A. y Montaño, E. (2011). La función Cobb Douglas en la producción de algodón del Valle de Juárez: Aplicación a factores definidos e interpretación específica de resultados, Revista Fuente, № 9, págs. 137-150.

Cubel, A. y Palafox, J. (2002). El Stock de Capital Productivo de la Economía Española, 1900 1990, Working Paper, IVIE.

Díaz, W. y Giral, D. (2011). Comparación de la solución análitica de la función de producción de Cobb Douglas con la obtenida por el método de mínimos cuadrados, Revista Tecnura, $\mathrm{N}^{\circ}$ 28, págs. 134-141.

Díaz; Z.; García, M. P. y Nuñez, J. (2013). Las funciones de Cobb-Douglas como base del espacio vectorial de funciones homogéneas, Universidad Complutense de Madrid, España.

Dixit, A. K., \& Stiglitz, J. E. (2004). Monopolistic competition and optimum product diversity, In S. Brakman \& B. J. Heijdra (Eds.), The monopolistic competition revolution in retrospect (pp. 70-88). New York: Cambridge University Press

Durán, J. (2015). Informe económico Políticas Económicas Aplicadas a Bolivia. Ministerio de Economía y Finanzas Públicas, (Viceministerio de Presupuesto y Contabilidad Fiscal), Bolivia.

Felipe, J. y Adams, F. (2005). The estimations of the Cobb Douglas funtion: a retrospective view, Eastern Economic Journal, Vol 31, № 3, págs. 427- 445. 
González, C.; Serva, R. y Tinoco, C. (2004). Estudio de factibilidad de logro de economías de escala en las fusiones bancarias venezolanas a través de la función Cobb-Douglas, Anales de la Universidad Metropolitana, Vol 4, № 1, págs. 111-126.

Humerez - Dorado (2004). Una aproximación de los determinantes del crecimiento económico en Bolivia 1960-2004. Unidad de análisis de políticas sociales y económicas. Análisis Económico, Vol 21, №3, págs. 45-60.

Ilca, M. y Popa, D. (2014). On approximate Cobb-Douglas production functions. Carpathian Journal of Mathematics, Vol 30, $\mathrm{N}^{\circ} 1$, págs. 87-92.

Jehle, G. A., y Reny, P. J. (2011). Advanced microeconomic theory (Tercera Edición). Harlow, England: Financial Times Prentice Hall.

Kumbhakar, S.C., S. Ghosh, and J.T. McGuckin (1991). A Generalized Production frontier Analysis, Approach for Estimating Determinants of Inefficiency in US Dairy Farms, Journal of Business and Economic Statistics, Vol 7, págs. 279-286.

López, C. y Palomares, D. (1999). Análisis de la función de producción agraria para distintos niveles de agregación, Estudios de Economía Aplicada, $\mathrm{N}^{\circ}$ 12, págs. 17-33.

Martínez G., A., y A. Castillo M. (1987). Teoría de la Regresión con Aplicaciones Agronómicas. Colegio de Postgraduados, Montecillo.

Montoya, O. y Soto, J. (2011). Estimación de la eficiencia técnica de las economías de los departamentos cafeteros de colombia aplicando la función Cobb Douglas translogarítmica con fronteras estocásticas y datos de panel, Scientia et Technica, Vol 1, № 47, págs. 8388 .

Morales, J. (2014). La economía boliviana de los últimos cincuenta años: reflexiones sobre el desarrollo de largo plazo, Ciencia y Cultura, № 33, págs. 107-135.

Muinelo-Gallo, L. (2012). Modelo estructural de función de producción. un estudio empírico de la innovación en el sector manufacturero español, Economía: Teoría y práctica, $\mathrm{N}^{\mathrm{o}} 36$, págs. 43-82.

Nombela, G. (2005). Infraestructura de transporte y productividad, Instituto de Estudios fiscalesUniversidad de las Palmas de Gran Canaria, España.

Parag, C.; Pendharkar, J. y Girish, H. (2008). An empirical study of the Cobb-Douglas production function properties of software development effort, Information and software technology, Vol 50, No 12, págs. 1181-1188.

Parra Osorio, Juan Felipe, (2012). Una función de producción para el teatro en Colombia. Cuadernos de Economía, Vol. 31, ํ5, págs. 153-187. 
Rajimon, J. (2010). La economía y la función de producción en educación. Revista Científica Visión de Futuro, Vol 13, N 1, págs. 31-43.

Ramírez Vigoya, A. (2015). Ajuste de una Función de Producción al Sector Financiero en Colombia. Revista Facultad de Ciencias Económicas: Investigación y Reflexión, Vol 23, N 1, págs. 141-156.

Rivera, W. (2016). Caso Bolivia: Del neoliberalismo hacia el modelo del pluralismo económico 1970-2015, Correo del Sur.

Sancho, A. (2004). Función de Producción Cobb-Douglas, Econometría de Económicas, págs. 2334.

Wang, X. y Fu, Y. (2013). Some characterizations of the Cobb-Douglas and CES production functions in microeconomics. Abstract and Applied Analysis, págs. 1-6.

Zurita Vaca, M., Amboya Soque, R. y Barba Castro, E. (2016). Infraestructura Vial y Crecimiento Económico: Caso Parroquias Sevilla Don Bosco y San Isidro, Provincia de Morona Santiago, Ecuador. Revista Investigaciones Altoandinas, Vol 18, № 1, págs. 76-89. 